Proceedings of the European Conference Physics of Magnetism 2011 (PM'11), Poznań, June 27-July 1, 2011

\title{
The Influence of Morphology on Magnetic Properties of Magnetosomes
}

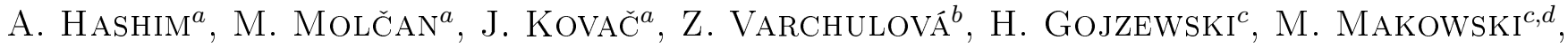 \\ P. KOPČANSKY ${ }^{a}$, Z. TOMORI ${ }^{a}$ AND M. TIMKO ${ }^{a}$ \\ ${ }^{a}$ Institute of Experimental Physics, SAS, Watsonova 47, 04001 Kosice, Slovakia \\ ${ }^{b}$ Institute of Medical Biology and Genetics, Faculty of Medicine UK, Sasinkova 4, 81108 Bratislava, Slovakia \\ ${ }^{c}$ Institute of Physics, Poznań University of Technology, Nieszawska 13A, 60-965 Poznań, Poland \\ ${ }^{d}$ Max Planck Institute for Polymer Research, Ackermannweg 10, 55128 Mainz, Germany
}

Cultivation process was changed three times. The culture medium was enriched by more amount ferric quinate (FQ magnetosomes sample) and more amounts Wolfe's vitamin solution (WVS magnetosomes sample) comparing to normal culture medium (NP magnetosomes sample). NP sample of magnetosomes shows zero coercivity and behaves superparamagnetically. The increase of coercivity (6.5 Oe for WVS and 20 Oe for FQ) may be caused by higher value of shape anisotropy and reveals stronger magnetic correlations between particles of magnetite chains.

PACS: $47.65 . \mathrm{Cb}, 51.60 .+\mathrm{a}, 75.30 . \mathrm{Gw}, 75.50 . \mathrm{Tt}, 75.60 . \mathrm{Nt}, 75.60 . \mathrm{Ej}$

\section{Introduction}

Magnetosomes are bacterial magnetic nanoparticles containing iron mineral crystals of magnetite $\left(\mathrm{Fe}_{3} \mathrm{O}_{4}\right)$ or greigite $\left(\mathrm{Fe}_{3} \mathrm{~S}_{4}\right)$. These are small in size of nanometer, disperse very well because they are covered with a stable biological membrane - phospholipid bilayer admixed with proteins. The morphology, size and intracellular organization of magnetosome crystals is subject to a species-specific biological control, which is genetically regulated by a complex and specific set of genes that have been identified within the magnetosome island within the genome of magnetotactic bacteria $[1,2]$. Magnetosomes are characterized by narrow grain-size distributions $(30-120 \mathrm{~nm})$, distinct species-specific crystal morphology, chemical purity, and arrangement in single or multiple linear chains [3]. Their function as a cellular compass to navigate along the geomagnetic field is well known $[4,5]$.

These structures are formed intracellularly in magnetotactic bacteria by a biomineralization process. The biomineralization process of magnetosomes is a complex, genetically controlled mechanism that controls the uptake, accumulation and precipitation of iron [6]. Magnetotactic bacteria are microorganisms that belong to a heterogeneous group of Gram-negative bacteria with diverse morphologies and habitats. They are a diverse group of aquatic prokaryotes [7]. In our experiment magnetosomes were synthesized by magnetotactic bacteria, $M a g$ netospirillum strain AMB-1 in laboratory conditions. In this contribution the morphology of magnetosomes obtained by various conditions during biomineralization process is given. The culture medium was enriched by more amount ferric quinate (FQ magnetosomes sample) and more amounts Wolfe's vitamin solution (WVS magnetosomes sample) comparing to normal culture medium (NP magnetosomes sample). The changes of cultivation process do not influence on magnetic properties of isolated magnetosomes. Magnetic nanoparticles in diluted aqueous suspensions are an important tool in medical diagnostics as contrast agent for magnetic resonance imaging and in therapy for magnetic drug targeting and hyperthermia.

\section{Experiments}

Bacterial magnetosomes were synthesized by biomineralization process of magnetotactic bacteria Magnetospirillum strain AMB-1.

The bacteria are a Gram-negative $\alpha$-proteobacterium that is more oxygen-tolerant bacteria. The medium for Magnetospirillum sp. AMB-1 consisted of (per $1 \mathrm{~L}$ medium): $10 \mathrm{~mL}$ Wolfe's vitamin solution, $5 \mathrm{~mL}$ Wolfe's mineral solution, $0.68 \mathrm{~g} \mathrm{KH}_{2} \mathrm{PO}_{4}, 0.848 \mathrm{~g}$ sodium succinate hexahydrate, $0.575 \mathrm{~g}$ sodium tartrate dihydrate, $0.083 \mathrm{~g}$ sodium acetate trihydrate, $0.225 \mathrm{ml} 0.2 \%(\mathrm{w} / \mathrm{v})$ resazurin (aqueous), $0.17 \mathrm{~g} \mathrm{NaNO}_{3}, 0.04 \mathrm{~g}$ ascorbic acid, $2 \mathrm{~mL} 0.01 \mathrm{M}$ ferric quinate [8]. Resazurin was added to media as colorimetric indicator of redox potential. The $\mathrm{pH}$ was adjusted to 6.75 with $\mathrm{NaOH}$. This medium was prereduced under nitrogen for a period of $1 \mathrm{~h}$, using copper as a reducing agent, and was subsequently dispensed into culture bottles in an anaerobic hood. Inoculated culture bottles were incubated at $25^{\circ} \mathrm{C}$ for a period of 10-14 days. In order to improve cultivation process the culture medium was changed by adding more amount FQ and more amount WVS comparing to normal culture medium. Techniques for the isolation of magnetosome particles from Magnetospirillum species are based on magnetic separation [9] or a combination of centrifugation and a magnetic separation technique. These procedures leave the surrounding membrane intact and magnetosome preparations are apparently free of contaminating material. 
The morphological properties and size of magnetosomes were estimated from scanning electron microscopy (SEM) and transmission electron microscopy (TEM). The micrographs of magnetosomes were obtained by low-voltage scanning electron microscope (LEO 1530 Gemini, Carl Zeiss NTS, Oberkochen, Germany) and TEM using JEOL1200EX microscope working at $120 \mathrm{kV}$. The samples for TEM experiments were prepared on amorphous carbon foil by micropipette of diluted aqueous solution of magnetosomes. The solution (solvent) was left to dry completely at room temperature. Samples for SEM were prepared by spin coating (Süss MicroTec, model Delta $80 \mathrm{BM}$ ) and drop coating deposition on polished silicon wafers (Grinm Semiconductor Materials Co., Ltd.).

The particle size and its distribution were analyzed by Ellipse 2.08 image processing program supplied with segmentation modules "Threshold" and "GraphCuts" (TEM micrographs). The whole process of analysis consists of three steps - calibration, segmentation and calculation of size (more detail is given in our work [10]).

Magnetization measurements of the prepared magnetosomes suspension were carried out by SQUID magnetometer of Quantum Design in magnetic field up to $5.5 \mathrm{~T}$ at room temperature.

\section{Experimental results}

Typical electron micrograph of magnetosomes on surface obtained by SEM technique is shown in Fig. 1. The whole process of analysis of size distribution from TEM photographs for every prepared sample consists of three steps - calibration, segmentation and calculation of size [10]. The result of segmentation is a set of polygons representing particles contours. Calculation of the size parameters from the segmented contours is the final step. Feret diameter, defined as the diameter of a circle having the same area as the object, was considered the most important parameter representing the size. Objects having very small compactness or extremely small area evidently represent artifacts and therefore they were removed from the statistics. For evaluation of different preparation conditions the size distributions of magnetosomes (from 100 particles) according to TEM photographs were prepared. The diameter of magnetosomes was estimated according to above mentioned method. The size distribution of magnetosomes obtained by cultivation at normal culture (NP), containing more amount FQ and more amount WVS are given in Fig. 2a-c, respectively. The mean diameter of magnetosomes isolated from new bacterial tin at normal culture medium $47 \mathrm{~nm}$ (Fig. 2a).

On the opposite side after adding more FQ or WVS the value of mean diameter is shifted to higher values $52 \mathrm{~nm}$ for WVS (Fig. 2b) and $58 \mathrm{~nm}$ for FQ (Fig. 2c). Very important was that cultivation process after adding WVS was shortening to 3-5 days and another fact that number of magnetosomes around the mean diameter was increased and distribution is narrower. Adding more quantity of $\mathrm{FQ}$ into the culture medium cultivation process

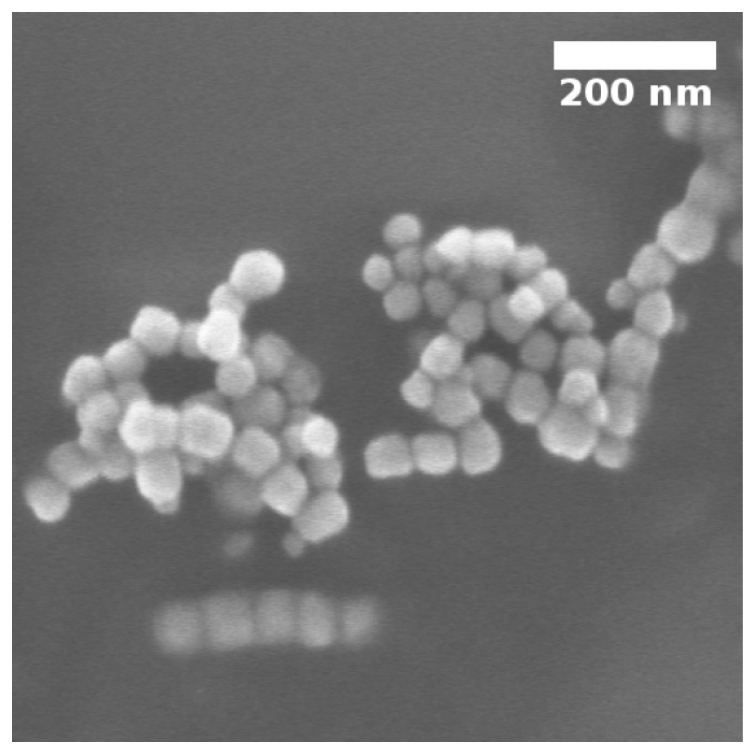

Fig. 1. Scanning electron micrograph of magnetosomes.

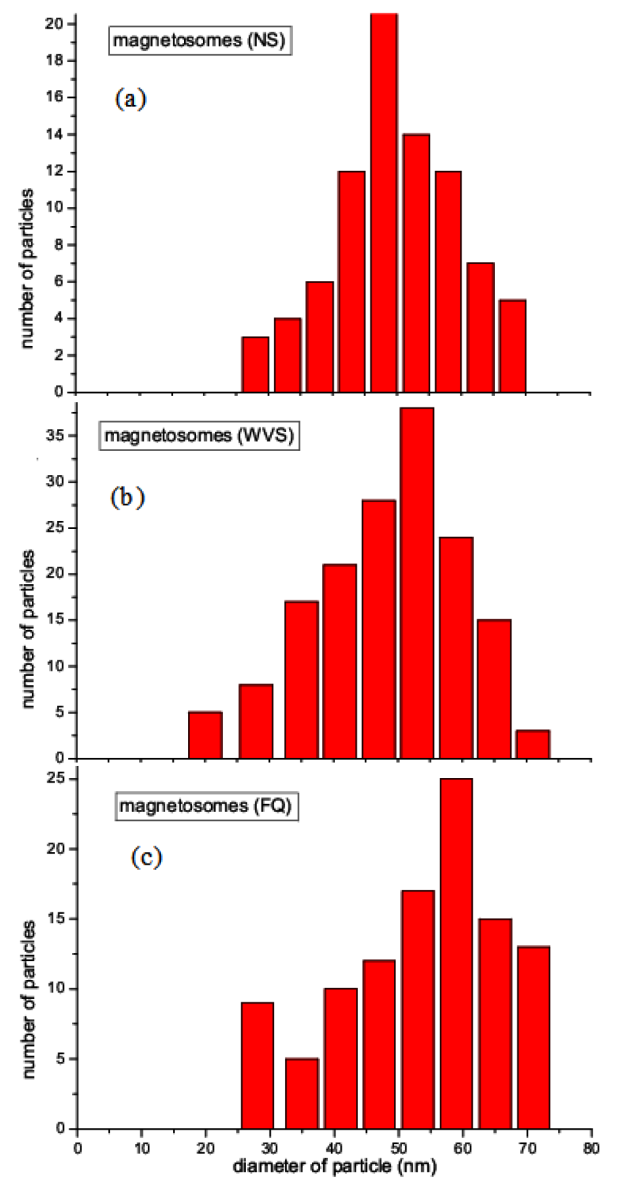

Fig. 2. Size distribution of magnetosomes obtained from different culture medium: normal (a), higher amounts of WVS (b), and higher amounts of FQ (c). 
was extended for roughly 21 days. There was observed increased number of magnetosomes in part of higher and lower size of magnetosomes which causes distinct changes of size distribution and size of magnetosomes is more uniform. Increased amount solution of FQ at the cultivation medium causes averaging size of obtained magnetosomes and mean size diameter is moved to greatly higher value (Fig. 2c).

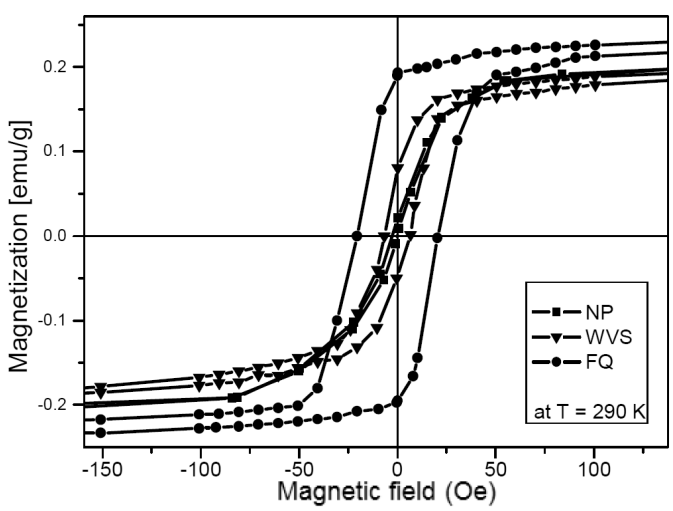

Fig. 3. Magnetization of the suspension of magnetosome versus magnetic field at $293 \mathrm{~K}$ obtained under NP (normal condition), with more amount WVS and FQ.

The curves of field dependence of magnetization at $293 \mathrm{~K}$ are reported in Fig. 3. These curves shows that zero hysteresis loop $\left(H_{\mathrm{c}}=0 \mathrm{Oe}\right)$ exists at room temperature on suspension of magnetosomes prepared at normal condition (NP) and this suspension behaves superparamagnetically. Small increase of hysteresis is observed for sample WVS $\left(H_{\mathrm{c}}=6.5 \mathrm{Oe}\right)$ and for sample FQ $\left(H_{\mathrm{c}}=20 \mathrm{Oe}\right)$ which means that these samples show ferromagnetic behavior. The data obtained from magnetization measurements are collected in Table.

\section{TABLE}

The mean diameter of magnetosomes $d$, coercive force $H_{\mathrm{c}}$ and saturation magnetization $M_{\mathrm{s}}$ of magnetosome suspensions prepared at various conditions.

\begin{tabular}{c|c|c|c}
\hline \hline & NP & WVS & FQ \\
\hline$d[\mathrm{~nm}]$ & 47 & 52 & 58 \\
$H_{\mathrm{c}}[\mathrm{Oe}]$ & 0 & 6.5 & 20 \\
$M_{\mathrm{s}}[\mathrm{emu} / \mathrm{g}]$ & 0.2 & 0.21 & 0.23
\end{tabular}

The reason may be caused by higher shape anisotropy, resulting in a higher coercivity for samples with higher mean diameter. The larger $H_{\mathrm{c}}$ value reveals stronger magnetic correlations between particles of magnetite chains.

\section{Conclusion}

The present result provides a synthesis method of magnetosome chains at various cultivation conditions.
The sizes of biological magnetite nanoparticles (magnetosome) was increased from $47 \mathrm{~nm}$ (NP) up to $52 \mathrm{~nm}$ (WVS) and $58 \mathrm{~nm}$ (FQ) as a consequence cultivation at higher amount of Wolfe's vitamin solution or ferric quinate. The chains of magnetite behave superparamagnetically for sample cultivated at normal condition and display ferromagnetic properties at room temperature for sample WVS and FQ. The increase of coercivity for sample WVS and FQ is connected with higher shape anisotropy for samples with higher mean size diameter. The low values for coercivity is connected with the fact that magnetosomes are still single-magnetic domain particles. As specific steps involved in magnetosome biomineralization process are still under dispute, our contribution showed that the changes of cultivation conditions can change the duration of process, morphology of magnetosome and consecutively the magnetic properties. So this type of research can offer some useful information to understand the biomineralization process.

\section{Acknowledgments}

This work was supported by Slovak Academy of Sciences, in the framework of CEXNANOFLUID, projects VEGA 0077, 0051, 0164 and Ministry of Education Agency for structural funds of EU in frame of projects Nos. 26220120021, 26220120033 and 26220220005. This work was supported by the Slovak Research and Development Agency under the contract Nos. APVV-0171-10, APVV-0682-07 and Sk-Pl 0069-09.

\section{References}

[1] S. Schübbe, M. Kube, A. Scheffel, C. Wawer, U. Heyen, A. Meyerdierks, M. Madkour, F. Mayer, R. Reinhardt, D. Schüler, J. Bacteriol. 185, 5779 (2003).

[2] S. Ullrich, M. Kube, S. Schübbe, R. Reinhardt, D. Schüler, J. Bacteriol. 187, 7176 (2005).

[3] D.A. Bazylinski, A.J. Garratt-Reed, R.B. Frankel, Microsc. Res. Technol. 27, 389 (1994).

[4] D. Faivre, D. Schueler, Chem. Rev. 108, 4875 (2008).

[5] A. Komeili, Ann. Rev. Biochem. 76, 351 (2007).

[6] S. Schübbe, M. Kube, A. Scheiffel, C. Wawer, U. Heyen, A. Meyerdierks, M.H. Madkour, F. Mayer, R. Reinhardt, D. Schüler, J. Bacteriol. 185, 5779 (2003).

[7] T. Matsunaga,T. Sakaguchi, Molecular mechanism of magnet formation in bacteria, Department of Biotechnology, Tokyo University of Agriculture and Technology Koganei, Japan 2000, p. 184.

[8] M.J. Wolin, E.A. Wolin, R.S. Wolfe, J. Biol. Chem. 238, 2882 (1963).

[9] K. Grünberg, C. Wawer, B.M. Tebo, D. Schüler, Appl. Environ. Microbiol. 67, 4573 (2001).

[10] H. Gojzewski, M. Makowski, A. Hashim, P. Kopcansky, Z. Tomori, M. Timko, Scanning 33, 1 (2011). 